\title{
Do Hydrodynamic Dispersion Coefficients Exist?
}

\author{
C.P. Lowe $\mathrm{e}^{1,2}$ and D. Frenkel ${ }^{1}$ \\ ${ }^{1}$ FOM Institute for Atomic and Molecular Physics, Kruislaan 407, 1098 SJ Amsterdam, The Netherlands \\ ${ }^{2}$ Computational Physics, Faculty of Applied Physics, Delft University of Technology, Lorentzweg 1, 2628 CJ Delft, The Netherlands
}

(Received 22 August 1996)

\begin{abstract}
We have calculated dispersion coefficients for tracer particles in a fluid flowing through a porous medium consisting of randomly packed spheres. At high Péclet numbers, where the motion of the tracer is determined largely by convection, we found evidence that the dispersion coefficient is diverging and that the dispersion process is anomalous. A transient region of anomalous dispersion has been predicted theoretically. However, our simulations suggest that, rather than being transient, this effect persists. We argue that our findings are consistent with the available experimental data. [S0031-9007(96)01712-7]
\end{abstract}

PACS numbers: 47.55.Mh, 05.60.+w

Tracer dispersion is the simplest example of the flow of an inhomogeneous two-component mixture. We assume that the two components, the "tracer" and the "solvent," have identical behavior but are distinguishable in the sense that they are assigned different "colors." The model may be simple, but it is neither trivial nor irrelevant. For instance, the solvent can represent a pure fluid and the tracer a contaminant. If the solvent occupies the space within some porous medium and is stationary, the tracer has a diffusion coefficient somewhat less than its value in the pure solvent (the latter we denote as $D_{0}$ ). If, however, the solvent flows through the porous medium with some mean flow velocity $\overline{\mathbf{v}}$, then, depending on the magnitude of $\overline{\mathbf{v}}$, the dispersion coefficient $D$ (defined as the tracer diffusion coefficient in a frame of reference moving with the mean flow velocity) can be very much greater than $D_{0}$. Understanding this phenomenon of "hydrodynamic dispersion" [1] is obviously important for modeling pollutant transport in ground water.

Hydrodynamic dispersion arises when the variations in the local fluid velocity spread the tracer over a larger volume than one would expect by diffusion alone (this is nicely illustrated in Ref. [2]). The Péclet number $P_{e}$ is a measure of the relative importance of diffusion and convection. It is defined as $P_{e} \equiv U^{*} l^{*} / D_{0}$, where $U^{*}$ and $l^{*}$ are, respectively, a characteristic velocity and a characteristic length scale. At high values of $P_{e}$, tracer transport over distances larger than $l^{*}$ is dominated by convection, and dispersion is dominated by the variations in the local fluid velocity.

With the notable exception of the Taylor-Aris result for a fluid flowing through a tube [3], there are few analytic results for $D$. A phenomenological argument [4] is that, at high Péclet numbers, molecular diffusion in porous media ceases to play a role and that dispersion is determined solely by spatial variations in the fluid velocity. In this limit $D / D_{0}=\alpha P_{e}$. The constant, $\alpha$, characteristic of a given medium, is known as the dispersivity. This result is derived using the Taylor hypothesis [5] which states that the dispersion coefficient equals the covariance of the velocity variations multiplied by a correlation time $\tau_{v}$. For packed spheres, $\tau_{v}$ is typically taken to be the time it takes to convect over a particle diameter. There is experimental evidence that, at least for a medium consisting of randomly packed spheres, this description is adequate for Péclet numbers ranging from $3 \times 10^{2}$ to $1 \times$ $10^{5}[4,6]$. The dispersivity has a value of $\alpha=1.8 \pm 0.4$ $[6,7]$. Because of its apparent success on the "laboratory" scale the phenomenological approach forms the basis for most models of dispersion on much larger scales [8].

Apart from the difficulty of predicting dispersivities, the physics of tracer dispersion at high Péclet numbers is, it seems, well understood. Below, we argue that this is not the case. Some evidence that there is a problem comes from experiment. According to the Taylor view, the dispersion coefficient should reach an effectively constant value in the time it takes a tracer to convect over a correlation length of the porous medium (typically of the order of a few particle diameters). However, experimentally $D$ is still found to increase on a much longer time scale $[9,10]$. Theoretical approaches which treat the solid-fluid interface more explicitly also suggest that things might not be quite so simple [11-13]. Koch and Brady [12] showed that for an isolated sphere (i.e., in the dilute limit) correlations in the fluid velocity decay so slowly that the hydrodynamic dispersion coefficient diverges [12]. That is, the mean square displacement, $\Delta(t)$, grows as $\Delta(t) \sim t \ln (t)$, rather than linearly in time. Koch and Brady, however, went on to assume that away from the dilute limit the velocity field far from the surface of a given sphere is given by the Brinkman equation. This is a mean-field theory which predicts that correlations in the velocity decay rapidly at distances greater than the square root of the permeability (the Brinkman length). For dense beds of spheres this is a small fraction of a particle radius. This "screened" decay is rapid enough for the dispersion coefficient to be finite. However, there should exist a boundary layer in which the velocity field which gives rise to the divergence of $D$ in the dilute limit persists. For the dispersion coefficient to reach its 
asymptotic value there must be time for the tracer to diffuse into (or out of) this layer, the width of which scales as $P_{e}^{-1 / 3} d$ ( $d$ is the sphere diameter). On shorter time scales the anomalous dispersion should persist [13]. This would have three important consequences. First, the dependence of $D$ on the Péclet number becomes $D / D_{0} \sim P_{e} \ln P_{e}$. Second, there should be a time for which the dispersion is anomalous. Third, rather than becoming irrelevant at high Péclet numbers, the molecular diffusion coefficient actually determines the time scale on which the dispersion coefficient converges. In this Letter we describe the results of computer simulations of hydrodynamic dispersion in random arrays of spheres. We restrict ourselves to the following questions: How valid is the Taylor hypothesis? And can we find evidence for the transient anomalous dispersion predicted by Koch and Brady [12]?

In our simulations we calculated the velocity autocorrelation function (VACF) for tagged particle motion in a flowing fluid. The VACF, which we denote $C_{v}(t)$, was calculated along the flow direction and in a frame of reference moving with the velocity, $\overline{\mathbf{v}}$. We therefore have $C_{v}(t)=\langle[v(0)-\bar{v}][v(t)-\bar{v}]\rangle$, where $v(t)$ is the component of the particle velocity parallel to $\overline{\mathbf{v}}$. It is convenient to introduce a time-dependent dispersion coefficient, $D(t) \equiv \int_{0}^{t} d t^{\prime} C_{v}\left(t^{\prime}\right)$. The dispersion coefficient, if it exists, is given as the limit of $D(t)$ for $t \rightarrow \infty$.

The fluid was described by a lattice Boltzmann model. This is a preaveraged version of a lattice gas [14], in which the state of the fluid is specified by the average number of particles, $n_{i}(\mathbf{r}, t)$, with velocity $\mathbf{c}_{i}$, at each site $\mathbf{r}$. The time evolution of the distribution functions is described by the discretized analog of the Boltzmann equation [15]. Tagged particle motion in the lattice Boltzmann system can be treated as follows. The probability that a particle moves with a velocity $\mathbf{c}_{i}$ after a collision, which we denote as $p_{i}(\mathbf{r})$, is given by $n_{i}(\mathbf{r}) / \rho(\mathbf{r})$, where $\rho(\mathbf{r})$ is the total number of particles at the node (here we consider a fluid which is in a steady state so there is no time dependence). Once we know $p_{i}(\mathbf{r})$ it is possible to calculate the VACF averaged over all possible tagged particle trajectories using the moment propagation method [16]. This has been described elsewhere [17].

Our calculations proceeded as follows. Having generated an appropriate solid geometry we applied a constant force density to the lattice Boltzmann fluid. Once the flow reached a steady state, we calculated $D(t)$. We tested the scheme against the few known analytic results and found it to reproduce accurately, even with a relatively crude lattice, both the time dependence and the value of the dispersion coefficient. We also calculated $D(t)$ for a simple cubic array of spheres where we can compare with the experimental results of Gunn and Pryce [18]. We found somewhat better agreement than that reported by Salles et al. [19]. A detailed description of this will be given elsewhere; here we are primarily interested in the randomly packed spheres. Configurations of spheres, with a solid volume fraction of 0.45 , were generated by a Monte Carlo simulation of a hard sphere fluid. This volume fraction is somewhat lower than that of the beds used experimentally. Salles et al. [19] reported only a weak dependence of the dispersivity on the volume fraction (at least at high densities). We also found this to be the case so we expect to be able to make a meaningful comparison with experiment. At the faces of the simulation box we applied periodic boundary conditions. However, in all cases we were careful to eliminate the effects of the periodic boundary conditions on the VACF by comparing data for various system sizes. At high Péclet numbers it was only possible to exclude the influence of the periodic boundary conditions up until the time required for particles to convect, on average, one quarter of the box length. This in itself suggests that correlations in the fluid velocity persist beyond the Brinkman length. The simulations were performed in a rectangular box with lengths 28 particle diameters (parallel to the flow direction) and 10 particle diameters (perpendicular to the flow direction), a particle diameter being 5 or 9 lattice units. The lattice spacing corresponds to just less than the Brinkman length for the particles of diameter 5 and just more than the Brinkman length for particles of diameter 9. However, the dispersion coefficients calculated using the different sizes of sphere differed by only a few percent and both gave the same time dependence for $D(t)$.

Figure 1 shows a plot of the dispersion coefficient as a function of the Péclet number. The Péclet number was defined with the velocity equal to the mean fluid velocity and the length equal to the particle diameter. We have also plotted experimental values measured on similar systems by Pfannkuch [6]. Both the simulations and experiments were conducted at low Reynolds numbers. By using the moment propagation technique the errors in the VACF,

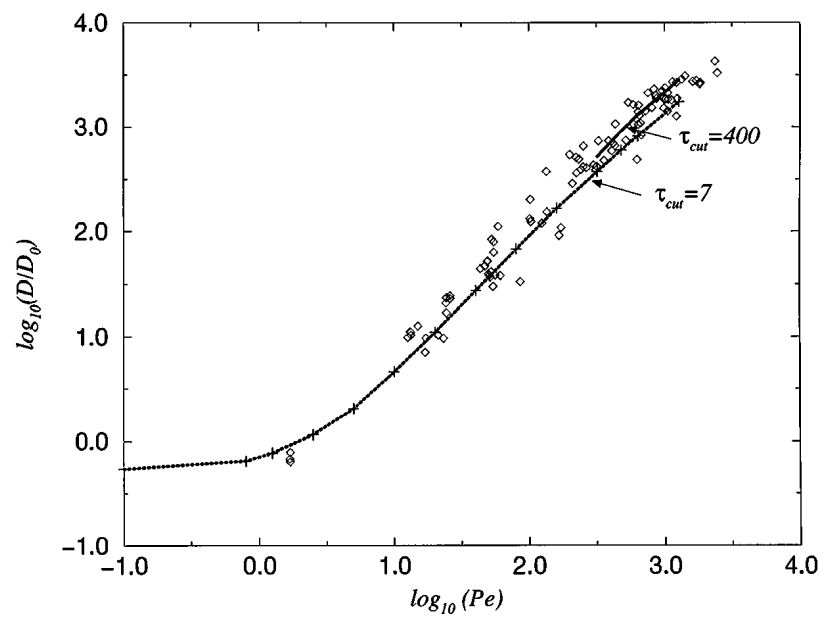

FIG. 1. Hydrodynamic dispersion coefficients, $D$, as a function of the Péclet number, $P_{e}$. The dotted line is a guide to the eye through the (uncorrected) simulation results (crosses). The diamonds are the experimental results of Pfannkuch [6]. The solid line shows the simulation results corrected by extrapolating the "tail" effect to experimental time scales. 
for a given configuration, are essentially zero. We do, however, need to average over a number of different configurations of spheres which introduces a statistical error. The number of configurations we used depended on the Péclet number, ranging from a minimum of 5 (at the lowest Péclet numbers) to a maximum of 24 (at the highest). For the values shown in Fig. 1 the error due to ensemble averaging is at most 5\%. A second source of error comes from integrating $C_{v}(t)$ up to a time $t_{\text {cut }}$ rather than over all times. At low Péclet numbers $\left(P_{e} \leq 80\right)$ the VACF has decayed to less than $2 \%$ of its initial value by $t=t_{\text {cut }}$. The error incurred by truncating the integral should therefore be small (unless, of course, the integral diverges). This truncation of the integral becomes more problematic at high Péclet numbers (see Fig. 2). However, we should bear in mind that the experimental dispersivities are also measured in a finite time interval. Although our measurements of $D$ are obtained by truncating the time integral at what is, by experimental standards, a short time, Fig. 1 shows that our numerical values for $D$ agree rather well with experiment. Our value for the dispersivity at the highest Péclet number we studied $\left(P_{e}=1280\right)$ is 1.35 . This is towards the lower end of the experimental range, $\alpha=1.8 \pm 0.4$. The dispersivity, $\alpha$, is not yet constant but is still increasing slightly with the Péclet number. This is despite the fact that we are well into the regime where dispersion is commonly assumed to be dominated by convection.

The VACF at higher Péclet numbers is shown in Fig. 2. The dimensionless time, $\tau$, is the time divided by the average time required for the tracer to convect a particle diameter. If the Taylor hypothesis is correct then the VACF should become independent of $D_{0}$ at high Péclet numbers; i.e., the curves shown in Fig. 2 should collapse onto a single curve. On going from $P_{e}=640$ to $P_{e}=$ 1280 the curves are, however, still distinguishable. There

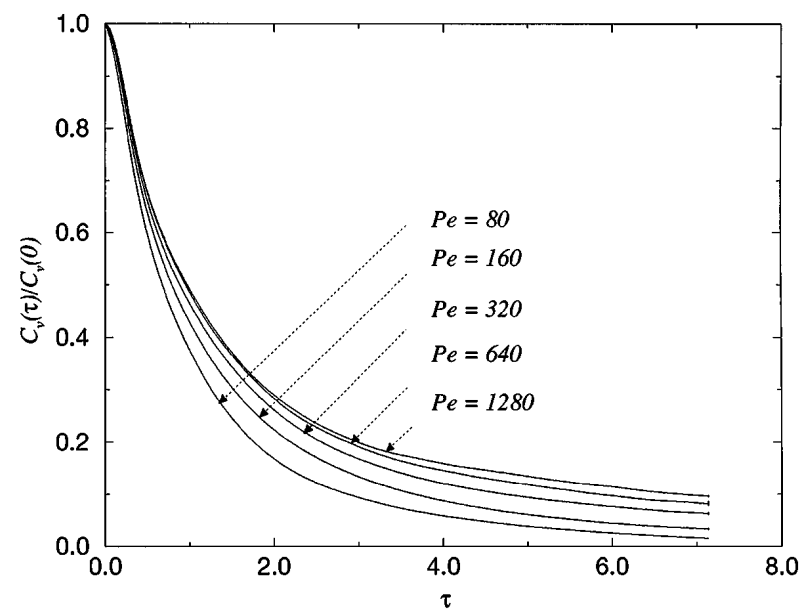

FIG. 2. The velocity autocorrelation function, $C_{v}(t)$, at various (high) Péclet numbers, plotted as a function of the reduced time $\tau$. are two further points to note from Fig. 1. First, at high Péclet numbers we are starting to miss a significant part of the integral by only integrating up to $\tau_{\text {cut }}$. This means that our values for $D$ are underestimated. Second, the decay of the VACF is not the exponential characteristic of hydrodynamic dispersion in simple geometries. In particular, the initial rate of decay is rather slow. A detailed analysis shows that it is in fact proportional to $D_{0}$ - even at very short times molecular diffusion is playing a role.

We now move to our second question, can we find evidence for transient anomalous dispersion? In Fig. 3 we have plotted the VACF multiplied by $\tau$. If the dispersion is anomalous the VACF decays as $1 / t$ and the plot should show a plateau. For Péclet numbers $P_{e} \geq 480$ we see that the VACF is indeed decaying as $1 / t$. The existence of this region of anomalous dispersion is in agreement with the theoretical prediction of Koch and Brady [12,13]. However, we find no evidence that this $1 / t$ decay is only transient. The Koch and Brady prediction is that the $1 / t$ decay persists only for a time proportional to $P_{e}^{1 / 3}$, after which the decay should be more rapid. We find that at $P_{e}=320$ the decay is more rapid than $1 / t$, but appears to approach a $1 / t$ decay at longer times. However, when increasing the Péclet number by only a factor of 1.5 (to $\left.P_{e}=480\right)$ we find that $1 / t$ decay persists right up until $\tau_{\text {cut }}$. At higher Péclet numbers we find evidence for only $1 / t$ decay. The fact that the anomalous dispersion persists once it appears seems more consistent with it being the true asymptotic behavior, rather than a transient effect that scales weakly with the Péclet number.

There is a way to exclude the possibility that the dispersion coefficient is diverging. Our simulations cover a time scale that, experimentally, is very short. We can, however, take our data and extrapolate the value of the dispersion coefficient to experimental time scales, assuming that the

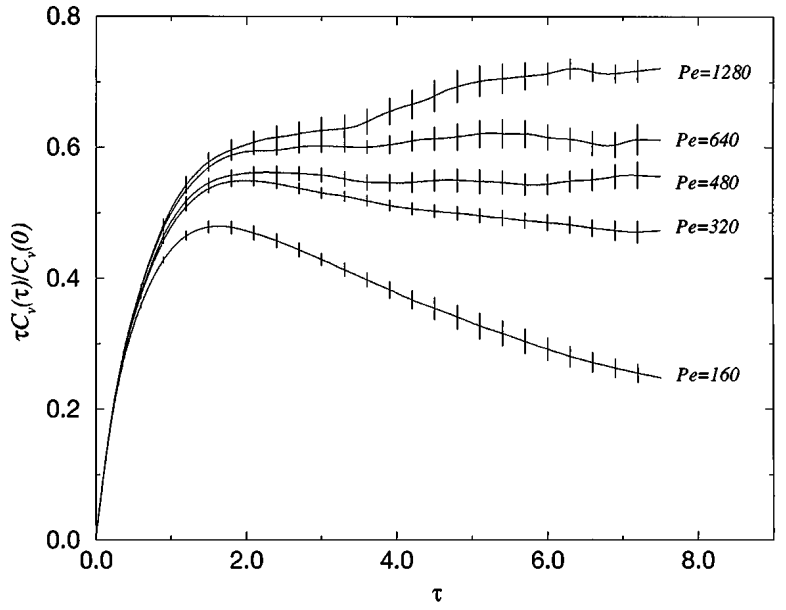

FIG. 3. The velocity autocorrelation function, $C_{v}(t)$, at various (high) Péclet numbers, multiplied by the reduced time $\tau$ and plotted as a function of $\tau$. A plateau in this plot means that $C_{v}(t)$ is decaying as $1 / t$. 
$1 / t$ decay persists. If this procedure leads to an inconsistency, we could conclude that the $1 / t$ decay must disappear on longer time scales than those accessible in the simulation. In the experiments performed by Pfannkuch [6] the (dimensionless) cutoff time, $\tau_{\text {cut }}$, varied from 400 to 1500. Our extrapolated values of $D$ for $\tau_{\text {cut }}=400$ are also shown in Fig. 1. The important point to note is that the assumption that the $1 / t$ decay persists (and hence that $D$ diverges) leads to estimates for $D$ that are, at least, consistent with experiment (and even marginally better than if we simply ignore the $1 / t$ decay beyond $\tau_{\text {cut }}$ ). Our data suggest that, in the range $320 \leq P_{e} \leq 1280$, the tail effect leads to an increase of about $25 \%$ in the apparent dispersivity on going from $\tau_{\text {cut }}=400$ to $\tau_{\text {cut }}=$ 1500. Pfannkuch's data in this range gives $\alpha=1.5 \pm 0.2$ $\left(\tau_{\text {cut }}=400\right)$ and $2.2 \pm 0.1\left(\tau_{\text {cut }}=1500\right)$, an increase of some $50 \%$. This is subject to appreciable statistical error, although not as much as Fig. 1 might, at first sight, suggest ( $\alpha$ is obtained by averaging over all data points within the specified range of Péclet numbers; this reduces the error in $\alpha$ as compared to the error in the individual data points). We can at least conclude that this trend is in agreement with the simulation.

In conclusion, our simulations suggest that at high Péclet numbers there is at least a region of anomalous dispersion. This means that the Taylor hypothesis is incorrect and that the modified relation predicted by Koch and Brady is more appropriate. However, our results are more consistent with the conclusion that the hydrodynamic dispersion coefficient actually diverges. A detailed experimental study of the limiting behavior of $D(t)$ would clearly be desirable. We stress that there is no reason why a dispersion coefficient must exist - there are several limiting cases where it does not (in a two-dimensional fluid [17], in a turbulent fluid [20], and even, in the dilute limit, for hydrodynamic dispersion itself [12]). Moreover, there is experimental evidence that the dispersion coefficient often does not reach a constant in time. Explanations that have been put forward to account for this observation are that the medium has a fractal structure [21] or that the dispersion mechanism is locally nonlinear [22]. Our results provide a much simpler explanation.

We thank Professor D. Koch and Ignacio Pagonabarraga for their comments. The work of the FOM Institute is part of the scientific program of FOM and is supported by the Nederlandse Organisatie voor Wetenschappelijk Onderzoek (NWO). Computer time on the CRAY-C98/ 4256 at SARA was made available by the Stichting Nationale Computer Faciliteiten (Foundation for National Computing Facilities).

[1] C. S. Slichter, Water Supply Papers 14R, U.S. Geological Survey (1905).

[2] N. S. Martys, Phys. Rev. E 50, 335 (1994).

[3] G. I. Taylor, Proc. R. Soc. London A 219, 186 (1953).

[4] J. J. Fried and M. A. Combarnous, Adv. Hydr. Sci. 7, 169 (1971).

[5] G. I. Taylor, Proc. London Math. Soc. 120, 196 (1921).

[6] H. D. Pfannkuch, Rev. Inst. Fr. Petrol. 18, 215 (1963).

[7] N. E. Rifaï, W. J. Kaufman, and D. K. Todd, Sanitary Eng. Report No. 3, I.E.R. series 90, Berkley, CA (1956).

[8] D. A. Chin, J. Hydr. Eng. 112, 591 (1985).

[9] N. Han, J. Bhakta, and R. G. Carbonell, AIChE J. 31, 277 (1985).

[10] E. Charlaix, J. P. Hulin, and T. J. Plona, Phys. Fluids 30, 1690 (1987).

[11] P. G. Saffman, J. Fluid Mech. 6, 321 (1959).

[12] D. L. Koch and J.F. Brady, J. Fluid Mech. 154, 399 (1985).

[13] D. L. Koch and J.F. Brady, Chem. Eng. Sci. 42, 1377 (1987).

[14] U. Frisch, B. Hasslacher, and Y. Pomeau, Phys. Rev. Lett. 56, 1505 (1986).

[15] A. J. C. Ladd, J. Fluid Mech. 271, 285 (1994).

[16] D. Frenkel and M.H. Ernst, Phys. Rev. Lett. 63, 2165 (1989).

[17] C. P. Lowe and D. Frenkel, Physica (Amsterdam) 220A, 251 (1995).

[18] D. J. Gunn and C. Pryce, Trans. Inst. Chem. Eng. 21, 583 (1969).

[19] J. Salles, J.-F. Thovert, R. Delannay, L. Prevors, J.-L. Auriault, and P. M. Adler, Phys. Fluids A 5, 2348 (1993).

[20] J. K. Klafter, M.F. Shlesinger, and G. Zumofen, Phys. Today 49, No. 2, 33 (1996).

[21] T.A. Hewett, presented at the 61st Annual Technical Conference of Petroleum Engineers, New Orleans, Louisiana, 1996.

[22] J. P. Pascal, Physica (Amsterdam) 223A, 99 (1996). 